\title{
Prefrontal-Occipitoparietal Coupling Underlies Late Latency Human Neuronal Responses to Emotion
}

\author{
Stephan Moratti, ${ }^{1,2}$ Cristina Saugar, ${ }^{1}$ and Bryan A. Strange ${ }^{3}$ \\ ${ }^{1}$ Department of Basic Psychology I, Complutense University of Madrid, 28223 Pozuelo de Alarcón, Spain, and ${ }^{2}$ Laboratory of Cognitive and Computational \\ Neuroscience and ${ }^{3}$ Laboratory for Clinical Neuroscience, Centre of Biomedical Technology, Technical University of Madrid and Complutense University of \\ Madrid, 28040 Madrid, Spain
}

Enhanced late positive potentials (LPPs) evoked by highly arousing unpleasant and pleasant stimuli have been consistently observed in event-related potential experiments in humans. Although the psychological factors modulating the LPP have been studied in detail, the neurobiological underpinnings of this response remain poorly understood. Current models suggest that the LPP is a product of both an automatic facilitation of perceptual activity, as well as postperceptual processing under cognitive control. Here we applied magnetoencephalography (MEG) and beamformer analysis combined with Granger causality measures to provide a mechanistic account for LPP generation that reconciles these two models. We demonstrate that the magnetic homolog of the LPP, mLPP, is localized within bilateral occipitoparietal and right prefrontal cortex. Critically, directed functional connectivity analysis between these brain regions, indexed by Granger causality, demonstrates stronger bidirectional influences between frontal and occipitoparietal cortex for high arousing emotional relative to low arousing neutral pictures. Thus, both bottom-up and top-down accounts of the late latency response to emotion derived from psychological studies can be explained by a reciprocal codependency between activity in prefrontal and occipitoparietal cortex.

\section{Introduction}

Event-related potential (ERP) studies have consistently demonstrated that emotionally arousing (pleasant and unpleasant) pictures evoke a larger late positive potential (LPP) than neutral pictures in a window from 400 to $800 \mathrm{~ms}$ after picture onset (Cuthbert et al., 2000; Schupp et al., 2000; Dolcos and Cabeza, 2002; Keil et al., 2002; Hajcak and Olvet, 2008; Hajcak et al., 2009). This effect is maintained after multiple stimulus repetitions, despite a decrement in autonomic arousal (Codispoti et al., 2006a), and is insensitive to physical features of the visual scene (Bradley et al., 2007). Furthermore, arousal modulation is maintained when emotional stimuli are presented as distractors or when participants are engaged in a competing task (Codispoti et al., 2006b). Thus, these observations led to a suggestion that affective modulation of the LPP is an automatic response to motivationally salient stimuli that does not rely on voluntary evaluation of emotional content (Codispoti et al., 2006a; Hajcak et al., 2007).

However, recent evidence conflicts with this model by demonstrating that late positive potential magnitude is sensitive to

\footnotetext{
Received June 10, 2011; revised Sept. 21, 2011; accepted 0ct. 10, 2011.

Author contributions: S.M. designed research; S.M. and C.S. performed research; S.M., C.S., and B.A.S. analyzed data; S.M., C.S., and B.A.S. wrote the paper.

This work was supported by a Ramón y Cajal fellowship (S.M.), the Plan Nacional sobre Drogas (C.S.), and the I3 program (B.A.S.) of the Spanish Ministry of Science and Innovation. This work was also funded by the Programa del Grupo de Biociencia de la Comunidad Autonóma de Madrid MADR.IB (M06094501).

The authors declare no competing financial interests.

Correspondence should be addressed to Stephan Moratti, Universidad Complutense de Madrid, Departamento de Psicología Básica I, Facultad de Psicología, Campus de Somosaguas, 28223 Pozuelo de Alarcón (Madrid), Spain. E-mail: smoratti@psi.ucm.es.

DOI:10.1523/JNEUROSCI.2917-11.2011

Copyright $\odot 2011$ the authors $\quad 0270-6474 / 11 / 3117278-09 \$ 15.00 / 0$
}

manipulations requiring voluntary cognitive control. LPP modulation is reduced following instructions to attend to less arousing aspects of unpleasant pictures (Hajcak et al., 2009). The LPP is also attenuated by cognitive reappraisal manipulations, with this decrease correlating with reductions in self-reported arousal intensity (Hajcak and Nieuwenhuis, 2006). Critically, although the LPP is enhanced by nonattended emotional stimuli (Codispoti et al., 2006b), directed attention operates additively with emotion, such that attended emotional stimuli evoke the largest LPP augmentation (Ferrari et al., 2008).Together, studies testing the psychological factors influencing the arousal-modulated LPP suggest that this response reflects a combination of "bottom-up" and "top-down" mechanisms (Ferrari et al., 2008).

That LPP arousal modulation can be subject to cognitive "topdown" control suggests involvement of prefrontal cortical areas, i.e., a prefrontal modulation of posterior visual attention areas. However, ERP studies of the LPP employing source localization techniques (Keil et al., 2002; Schupp et al., 2007) have not reported prefrontal activation. By contrast, direct electrocortical stimulation of prefrontal cortex (PFC) during emotional picture presentation disrupts arousal modulation of the LPP over posterior electrode sites (Hajcak et al., 2010), lending support for a pivotal prefrontal role in LPP generation. Neuroimaging studies report prefrontal activation in response to emotional pictures (for review, see Phan et al., 2002), but fMRI lacks the temporal resolution to test for emotion-dependent activity specific to the LPP time window.

Here we adopt a novel approach to exploring emotional stimulus-evoked late latency neuronal responses. We hypothesized that the millisecond time resolution of magnetoencephalography 
(MEG) recordings, combined with source reconstruction techniques, would provide evidence for a specific prefrontal role in emotion-induced late latency modulation of neuronal responses. We first demonstrate a neuromagnetic counterpart of the affect modulated LPP (mLPP) in response to emotionally arousing pleasant and unpleasant pictures. Furthermore, beamformer source localization reveals that the mLPP is localized to a prefrontal-occipitoparietal network. Critically, as predicted by mechanistic accounts of LPP generation, the MLPP is associated with increased bidirectional functional connectivity between prefrontal and occipitoparietal cortex.

\section{Materials and Methods}

\section{Participants}

Twenty-three subjects (12 females; 20 right, three left handed, and one ambidextrous; Oldfield, 1971) aged 21-38 years (mean 27.8 years) participated in our study after giving written informed consent. All subjects had normal or corrected to normal vision. The study had full ethical approval.

\section{Stimuli and experimental design}

Stimuli comprised 60 color pictures selected from the International Affective Picture System or IAPS (Lang et al., 2005): 20 high-arousing pleasant (erotic couples and family scenes), 20 high-arousing unpleasant (mutilations and attack scenes), and 20 low-arousing neutral (household scenes and neutral persons) pictures. Mean normative valence ratings (SEM) on a 9-point scale were $7.33( \pm 0.13)$ for pleasant, $4.87( \pm 0.05)$ for neutral, and $2.76( \pm 0.19)$ for unpleasant pictures. Mean normative arousal ratings for pleasant, neutral, and unpleasant pictures on a 9-point scale were $5.44( \pm 0.24), 2.8( \pm 0.13)$, and $6.9( \pm 0.07)$, respectively.

Pictures were presented by video projector (JVC DLA-G11E) via a mirror system to the center of a screen in a magnetically shielded MEG room. All pictures subtended a visual angle of $10^{\circ}$ horizontally and vertically. A central fixation cross subtending a visual angle of $1.6^{\circ}$ horizontally and vertically was presented throughout the whole experiment. Subjects were instructed to fixate during picture presentation.

We employed the experimental design of Schupp et al. (2000). Pictures were presented for $1.5 \mathrm{~s}$ in sequences of six. Each sequence contained two pleasant, neutral, and unpleasant picture trials in random order. Subjects were instructed to indicate, at the offset of each stimulus, whether the picture was pleasant, neutral, or unpleasant by using three response buttons (response button assignment counterbalanced across subjects). The interstimulus interval varied randomly between 1.5 and 3 s. Each sequence of six pictures was followed by an interval of $3 \mathrm{~s}$. The first experimental block comprised 30 sequences and, after a 1 min break, the procedure repeated in a second block. Thus, each picture category (pleasant, neutral, and unpleasant) contained 120 trials.

After the MEG session, participants rated the same pictures, presented self-paced in random order, with respect to valence and arousal using the Self-Assessment Manikin scale (SAM; Lang et al., 2005).

\section{Data acquisition and preprocessing}

Each participant's head shape and five index points (nasion, left and right periauricular points, and two additional positions at the forehead) were digitized to obtain the head position relative to the MEG sensors. Two $\mathrm{Ag} / \mathrm{AgCl}$ electrooculogram (EOG) electrodes were attached near the left and right outer canthi and two above and below the right eye. An electrode at the right mastoid served as ground.

MEG data were recorded continuously and digitized at a rate of 254.3 $\mathrm{Hz}$ using a 148-channel whole head system (Magnes 2500 WHS, 4-D Neuroimaging). A bandpass filter of 0.1 to $50 \mathrm{~Hz}$ was applied online. EOG was recorded with a Synamps amplifier (Neuroscan) using $\mathrm{Ag} / \mathrm{AgCl}$ electrodes with the same sampling rate and online filter as with the MEG recordings. The raw MEG data were filtered offline $(0.1 \mathrm{~Hz}$ highpass with a $6 \mathrm{~dB}$ /octave slope and $30 \mathrm{~Hz}$ lowpass with a $48 \mathrm{~dB}$ /octave slope). For each MEG channel, artifact-free epochs with a prestimulus baseline of $0.5 \mathrm{~s}$ and a poststimulus interval of $0.8 \mathrm{~s}$ were averaged to obtain the stimulus-locked event-related field (ERF) for each subject and condition.
We did not extend the time window of interest beyond $800 \mathrm{~ms}$, as previous data using the same stimulus presentation parameters (Schupp et al., 2000) showed termination of LPP effects at 0.8 s. Epochs that contained eye blinks or eye movements as determined by the EOG, as well as incorrect trials, were discarded from our analyses. Furthermore, epochs with movement artifacts identified by visual inspection of the data were eliminated. Finally, epochs that contained channels with peak amplitude $>3$ pT were also considered artifacts and discarded.

\section{Sensor space}

Realignment of MEG data in the sensor space. Subject-specific sensor positions were projected onto standard MEG sensor locations as implemented in FieldTrip (http://fieldtrip.fcdonders.nl/) (Oostenveld et al., 2011)). Briefly, the MEG data were projected onto a current dipole set (600 dipoles) in a spherical head model and then projected back onto standard MEG sensors, the positions of which were determined by the mean Cartesian sensor coordinates across all subjects (Knösche, 2002).

Statistical assessment of affective ERF modulation. The realigned MEG ERF data were averaged between 0.4 and $0.6 \mathrm{~s}$ to obtain a grand mean topography across subjects corresponding to the electrophysiological LPP time window. The realigned ERF topographies were submitted to a nonparametric cluster-based permutation statistic (Nichols and Holmes, 2002; Maris and Oostenveld, 2007), as implemented in FieldTrip, to identify sensors of significant picture category effects. This test effectively controls the familywise error rate in the context of multiple comparisons (148 sensors) by clustering neighboring channels that exhibit the same effect. At each sensor, a multivariate Hoteling's $T^{2}$ was calculated at an initial parametric threshold of $p<0.01$ to obtain a measure for picture category effects. Based on this threshold, significant sensor clusters were formed by spatial adjacency (a cluster had to consist of at least two significant neighboring sensors). One thousand permutations were performed. For each permutation the maximum sum of $T^{2}$ values within one cluster entered into the permutation distribution (i.e., exceedance mass; see Poline et al., 1997). Finally, the cluster step was performed with the observed ERF data. Observed cluster sums of $T^{2}$ values that were greater than the 95th percentile $(p<0.05)$ of the permutation distribution were considered as sensor clusters of significant picture category effects. Mean ERF values across significant sensor clusters were further analyzed by simple comparisons using the least significant difference test to assess differences between each picture category. Furthermore, the grand mean ERF waveforms across subjects and sensor clusters for each time point were extracted to compare them with the LPP waveforms as reported in the ERP literature.

Furthermore, because different studies have reported slightly varying LPP time windows (especially with respect to the duration of the LPP effect; Schupp et al., 2007; Hajcak et al., 2010), we repeated our sensor space analysis for the $0.6-0.8 \mathrm{~s}$ poststimulus interval. No sensor clusters demonstrating a picture category effect were found even after lowering the initial parametric threshold to $p<0.05$. However, the ERF waveforms of the mLPP sensor clusters (see Fig. 1) suggested middle latency $(0.2-0.4 \mathrm{~s})$ emotion effects. Indeed, the "early posterior negativity" (EPN) is a well known arousal modulated ERP component (Schupp et al., 2007) that has its neuromagnetic counterpart (mEPN) in temporal sensor clusters (Peyk et al., 2008). Given that the focus of the present study is to characterize the mLPP, analysis of the mEPN is not included here. Thus, we limited all subsequent statistical and source space analyses to the $0.4-0.6 \mathrm{~s}$ interval poststimulus onset.

\section{Source reconstruction}

The underlying current source density of the magnetometer-based ERFs was estimated for each time point, picture category, and subject by a linearly constrained, minimum variance beamformer (Van Veen et al., 1997) as implemented in FieldTrip. A regularization parameter of $10 \%$ was used. A dipole mesh (7000 surface dipoles) derived from a template brain [Collin 27 template brain aligned with the Montreal Neurological Institute (MNI) phantom brain; Collins et al., 1998] was used to calculate the forward solution using a head model based on overlapping spheres (for each channel a local sphere was fitted to the underlying head shape points; Huang et al., 1999). Before calculating the forward solution, the 
head and sensor positions of each subject were coregistered with the template brain by realigning the individual with the template brain's fiducials and further refined by minimizing the mean distance between the individual head shape points and the template brain scalp surface.

\section{Statistical assessment of affect modulated cortical current source densities}

Current source densities (dipole moments) obtained by the beamformer were averaged across time intervals between 0.4 and $0.6 \mathrm{~s}$ (see above). Next, mean vectors of each subject and condition were normalized by their corresponding mean baseline ( $0.5 \mathrm{~s}$ prestimulus) to obtain relative power changes with respect to the prestimulus interval. The beamformer relative power changes of each participant and picture category were submitted to the same nonparametric cluster-based permutation statistic as described above to identify cortical source clusters of affect modulation. Based on sensor space results (see Results), a quadratic contrast specifically testing that high-arousing pleasant and unpleasant pictures generate greater power changes than low-arousing neutral pictures ( pleasant $=$ unpleasant $>$ neutral) was evaluated by a multivariate $T^{2}$ test. Dipole clusters were considered significant at a corrected threshold of $p<0.05$. At sensor level, the ERF waveforms of significant sensor clusters suggested that the middle latency effects $(0.2-0.4 \mathrm{~s} ; \mathrm{mEPN})$ captured by these sensors were probably due to volume conduction effects. However, at source level the observed source waveforms of significant dipole clusters were, theoretically, more specific to the mLPP time window, as volume conduction is accounted for by the beamformer. Therefore, we extracted the mean source waveforms (virtual electrodes) for each significant dipole cluster (see Fig. $4 B$ ) and averaged the waveforms across $0.2-0.4 \mathrm{~s}, 0.4-0.6 \mathrm{~s}$, and $0.6-0.8 \mathrm{~s}$ time intervals. For each dipole cluster, a MANOVA with picture category and time interval as withinsubject factors was calculated to test for the specificity of picture category effects for the mLPP interval $(0.4-0.6 \mathrm{~s})$. When this MANOVA indicated a picture category by time interval interaction, separate MANOVAs with picture category as a within-subject factor were calculated for each time interval separately. Simple comparisons were conducted where necessary.

\section{Directed functional connectivity analysis}

We employed the "Granger causal connectivity analysis" toolbox (http:// www.anilseth.com/) implemented in Matlab (Mathworks) (Seth, 2010). Briefly, multivariate autoregressive (MVAR) models were used to determine whether knowledge of time series A helps to predict time series B, i.e., whether A "Granger causes" B (Granger, 1969). The time series for each participant, condition, and dipole within each dipole cluster (as determined by the nonparametric cluster-based permutation statistics) were reconstructed using the spatial filters derived by the beamformer by multiplying the sensor data matrix by the spatial filters corresponding to the dipoles within the established clusters. Next, for each subject and condition a principal component analysis (PCA) was applied to all dipole time series within a dipole cluster (Zhou et al., 2009) for the mLPPrelevant time window $(0.4-0.6 \mathrm{~s})$. The first five PCA components were averaged so that information loss was $<5 \%$ (Zhou et al., 2009).

To ensure covariance stationarity (Seth, 2010), the temporal mean of the PCA-based time series for each ROI, subject, and condition were removed and the ensuing mean-corrected time series was tested using the KPSS (Kwiatkowski-Phillips-Schmidt-Shin) test (Kwiatkowski et al., 1992). All time series for the MLPP window fulfilled the prerequisite of covariance stationarity. In a next step, the model order for the MVAR model was determined according to the Bayesian information criterion (BIC; Schwarz, 1978). In MVAR models, the model order serves to "reduce" model space, as too many parameters would over-fit the data and too few would poorly represent the data. The BIC criteria balance the variance accounted for by the model against the number of model parameters. Across all participants and conditions the model orders ranged between 2 and 10. Finally, significant Granger causality (GC) interactions between dipole clusters were determined by an $F$ test for each subject and condition (Seth, 2010). Participants that showed no significant GC interactions in at least two of the three picture categories were excluded from our main Granger causality analyses.
Following Keil et al. (2009), two approaches were chosen to statistically compare the GC interactions between the three picture categories. First, the overall number of unidirectional and bidirectional significant interactions was entered into a MANOVA model with picture category as within-subject factor. Significant picture category effects were further analyzed by quadratic contrast analysis (pleasant $=$ unpleasant $>$ neutral). Second, the nonparametric McNemar test (McNemar, 1947) for paired samples was used to test differences between each picture category pair and for each dipole cluster combination. This analysis was done separately for significant unidirectional and bidirectional GC interactions.

Finally, based on arousal-dependent right fronto-occipitoparietal bidirectional connectivity (see Results), bidirectional GC arousal values (averaged GC values across pleasant and unpleasant minus neutral picture category) were compared between time windows of 0.2-0.4 s, 0.4$0.6 \mathrm{~s}$, and $0.6-0.8 \mathrm{~s}$ by applying a MANOVA model with time window as the within-subject factor. Polynomial contrast analysis was used to characterize the profile of the bidirectional arousal GC values across time. This was done to investigate the specificity of GC differences between picture categories for the mLPP time range. The $0.0-0.2 \mathrm{~s}$ time window was not included in this analysis, as the early peak of activity violated covariance stationarity (KPSS test; Kwiatkowski et al., 1992).

\section{Results \\ SAM ratings}

Valence ratings differed significantly across picture category $\left(F_{(2,20)}=225.5, \lambda=0.04, p<0.0001, \eta^{2}=0.95\right)$. Pleasant pictures were rated as most pleasant $(7.8 \pm 0.16)$, followed by neutral $(5.1 \pm 0.06)$ and unpleasant pictures $(2.0 \pm 0.12 ; p<$ 0.0001 for all simple comparisons). Arousal ratings also differed across picture category $\left(F_{(2,20)}=83.1, \lambda=0.10, p<0.0001, \eta^{2}=\right.$ $0.89)$. Pleasant and unpleasant pictures were rated as more arousing than neutral ones, while unpleasant pictures scored higher arousal ratings than pleasant ones (pleasant: $5.5 \pm 0.29$; unpleasant: $6.8 \pm 0.21$; neutral: $2.5 \pm 0.26 ; p<0.0001$ for all simple comparisons).

\section{Event-related fields}

The nonparametric cluster-based permutation statistics on our sensor-space data revealed two significant sensor clusters for the mean magnetic flux maps between 0.4 and $0.6 \mathrm{~s}$, indicating different neuromagnetic responses for each picture category. The 95th percentile critical value for the sum of $T^{2}$ values (exceedance mass) was 82.4. Clusters emerged across left temporal (exceedance mass: $\operatorname{sum}\left(T^{2}\right)=440, p<0.0001$ ) and right temporal (exceedance mass: $\operatorname{sum}\left(T^{2}\right)=487, p<0.0001$ ) sensors. Figure $1 A$ depicts the grand mean ERF waveforms across participants for left and right sensor clusters (Fig. $1 A$, insets), illustrating the time course of the neuromagnetic response. Both sensor cluster waveforms were characterized by a slow magnetic field change that is highly similar to the slow potential shifts typically observed during the LPP time window (Schupp et al., 2000).

ERF responses within both left and right temporal sensor clusters, averaged across the mLPP time window, were greater for pleasant and unpleasant pictures compared to neutral pictures (pleasant vs neutral, $p<0.001$, unpleasant vs neutral, $p<0.0001$ for both sensor clusters). In the right sensor cluster, unpleasant pictures evoked the biggest ERF amplitude (unpleasant vs pleasant, $p<0.01$; Fig. 1B), whereas on the left, pleasant and unpleasant pictures did not differ $(p=0.33$, Fig. $1 B)$.

These sensor-space results should, however, be viewed with the caveat that the underlying source of the neuromagnetic response is not readily obtained from topographical MEG flux maps derived from magnetometers. Thus, having demonstrated significant effects of emotion at the sensor level, we went on to 
A
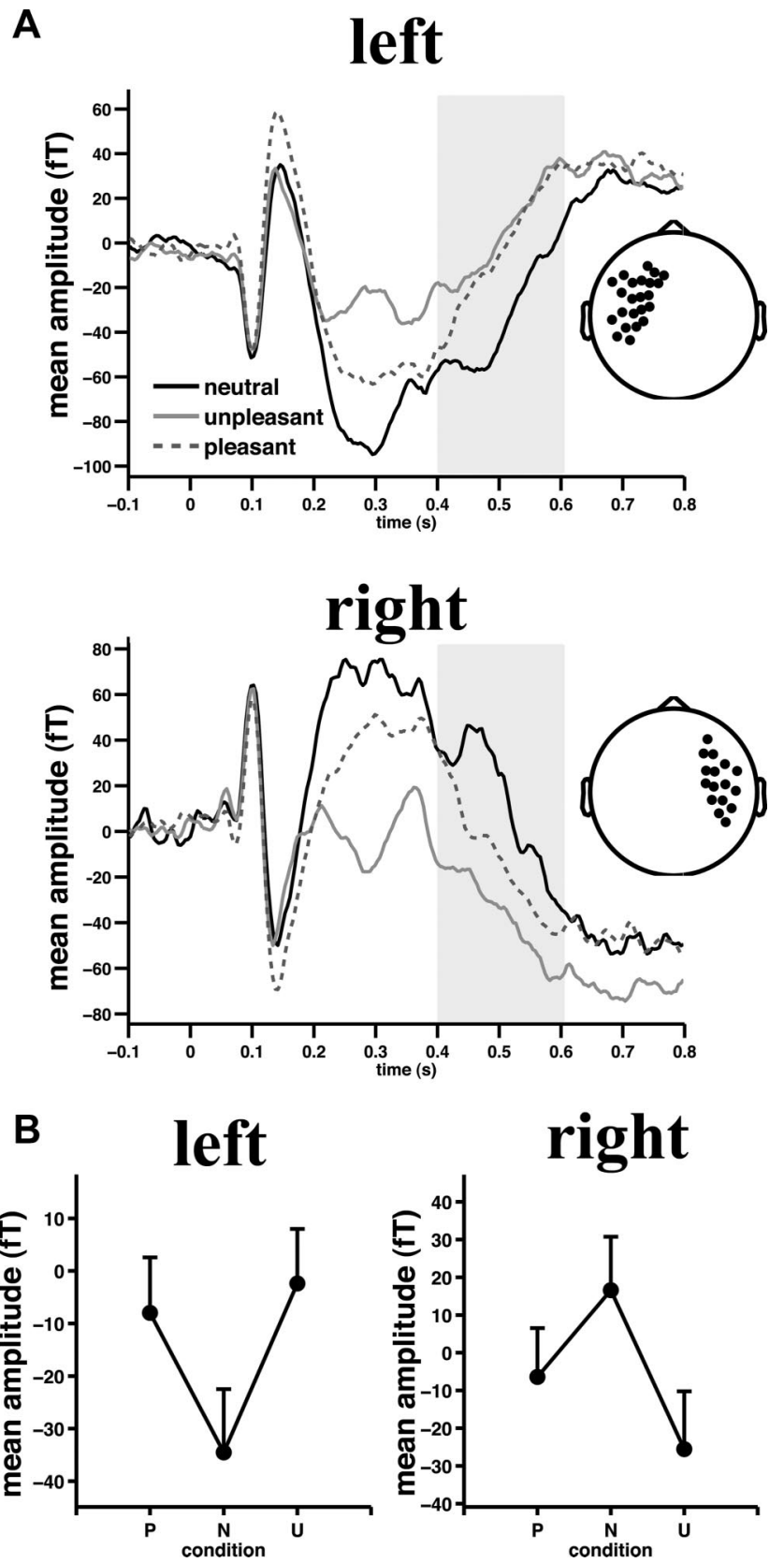

right

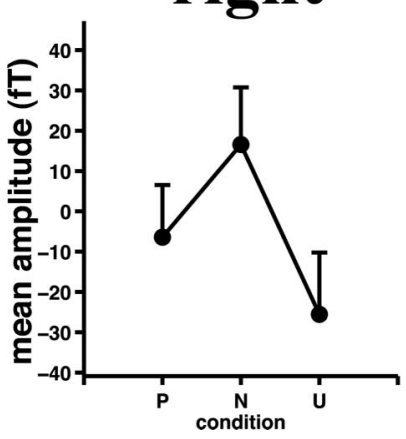

Figure 1. Significant emotional arousal modulation of sensor-level activity. $\boldsymbol{A}$, Grand mean ERF waveforms across left (top) and right (bottom) temporal sensors as detected by permutation statistics between 0.4 and $0.6 \mathrm{~s}$ (gray shaded area). Insets depict sensor cluster locations. Note that differences between 0.2 and $0.4 \mathrm{~s}$ most likely reflect the magnetic counterpart of the early posterior negativity also present in the temporal sensor clusters due to volume conduction. $\boldsymbol{B}$, Grand mean ERF amplitude across temporal sensors and the time interval of interest are shown. Positive versus negative values are due to reversed field directions. Pattern reversal of magnetic flux directions is due to different underlying cortical source orientations. Error bars, here and in subsequent figures, indicate SEM. P, Pleasant; $N$, neutral; $U$, unpleasant.

derive our anatomical inference on mLPP generators in source space.

\section{Beamformer analysis}

A difference map between source localized emotional (pleasant and unpleasant) and neutral responses is given in Figure $2 \mathrm{~A}$. The nonparametric cluster-based permutation statistics spanning a quadratic contrast (pleasant $=$ unpleasant $>$ neutral arousal effect) revealed two significant dipole clusters for mean relative power changes between 0.4 and $0.6 \mathrm{~s}$ at a familywise-corrected alpha level of 0.05 (critical exceedance mass $\operatorname{sum}\left(T^{2}\right)=491.5$; Fig. 2B, top). The most significant cluster (exceedance mass $\operatorname{sum}\left(T^{2}\right)=578.1, p<0.05$ ) was observed in right PFC (see Table 1 for MNI coordinates). A second cluster (exceedance mass $\operatorname{sum}\left(T^{2}\right)=497.3, p<0.05$, see Table 1) was localized in left occipitoparietal cortex. A third dipole cluster, in right occipitoparietal cortex, demonstrated arousal modulation with corrected significance at trend level (exceedance mass $\operatorname{sum}\left(T^{2}\right)=428.0$, $p=0.06$ ). To examine this network at a less stringent statistical threshold, we applied a parametric threshold to the empirical $T^{2}$ map. All three clusters were observed at an uncorrected threshold of $p<0.01$ (Fig. $2 B$, bottom); thus, these three regions were taken as the neuroanatomical network included in the GC analysis (see below, Granger causality analysis). Enhanced responses to emotional pictures in all clusters comprising this network (Fig. 2C) clearly demonstrate arousal modulation (a posteriori single comparisons between mean cluster activity showed no differences between pleasant and unpleasant pictures: all $p$ values $>0.43$; emotional pictures differed from neutral stimuli: all $p$ values $<0.02$ ). Note that regions of negative differences (pleasant $=$ unpleasant $<$ neutral), illustrated in blue in Figure $2 A$, did not reach corrected or uncorrected significance (Fig. $2 B$ ).

Figure $4 B$ shows the source waveforms of the three identified dipole clusters between 0.2 and 0.8 s poststimulus time. To demonstrate that activity in these dipole clusters specifically represent mLPP amplitude arousal modulation, we tested for picture category by time interval $(0.2-0.4 \mathrm{~s}, 0.4-0.6 \mathrm{~s}$, and $0.6-0.8 \mathrm{~s})$ interactions. This interaction was significant in all three brain regions; picture category effects differed across the three time intervals analyzed (left occipitoparietal cortex: $F_{(4,19)}=3.8, \lambda=0.55, p<$ $0.02, \eta^{2}=0.45$; right occipitoparietal cortex: $F_{(4,19)}=4.1$, $\lambda=0.54, p<0.02, \eta^{2}=0.46$; right PFC: $F_{(4,19)}=4.1, \lambda=0.54$, $\left.p<0.02, \eta^{2}=0.46\right)$. In all three brain regions, mean source activity only differed during the mLPP interval (left occipitoparietal: $F_{(2,21)}=7.7, \lambda=0.58, p<0.01, \eta^{2}=0.42$; right occipitoparietal: $F_{(2,21)}=7.1, \lambda=0.60, p<0.01, \eta^{2}=0.40$; PFC: $\left.F_{(2,21)}=11.6, \lambda=0.48, p<0.001, \eta^{2}=0.52\right)$. The mLPP changes were best described by arousal modulation (left occipitoparietal quadratic contrast: $F_{(1,22)}=14.8, p<0.001, \eta^{2}=0.40$; right occipitoparietal quadratic contrast: $F_{(1,22)}=9.0, p<0.001, \eta^{2}=0.40$; right PFC quadratic contrast: $\left.F_{(1,22)}=24.2, p<0.001, \eta^{2}=0.52\right)$.

Given that in our Granger causality analyses six subjects did not show reliable GC interactions (see below, Granger causality analysis), we verified the group-level arousal modulation effect in the remaining 17 subjects upon which the GC analysis was based. Mean dipole cluster contrast analysis demonstrates that the quadratic effect during the mLPP time window remains significant in all three clusters (left occipitoparietal: $F_{(1,16)}=17.6, p<0.001$, $\eta^{2}=0.52$ : right occipitoparietal: $F_{(1,16)}=6.2, p<0.001, \eta^{2}=$ 0.50 ; right prefrontal: $\left.F_{(1,16)}=16.2, p<0.001, \eta^{2}=0.50\right)$.

\section{Granger causality analysis}

We first examined GC interactions for each subject individually. Six subjects failed to show significant between-cluster interactions in more than one picture category and were therefore excluded from our main GC analysis. Thus, the results of directed functional connectivity are based on 17 participants ( 10 female), who showed reliable GC interactions for at least two picture categories. Across all conditions and 17 participants, $38 \%$ of all possible connections were significant.

Higher numbers of bidirectional GC interactions between all clusters during viewing of pleasant and unpleasant relative to neutral 
pictures were observed $\left(F_{(2,15)}=11.3, \lambda=\right.$ $0.39, p<0.001, \eta^{2}=0.60$; quadratic contrast $F_{(1,16)}=23.7, p<0.0001, \eta^{2}=0.60$; mean number of bidirectional connections for pleasant: $1.11 \pm 0.34$; neutral: $0.35 \pm$ 0.15; unpleasant: $1.59 \pm 0.31$; Figure $3 A$ ). Note that including all subjects (i.e., including the six subjects with unreliable GC interactions) did not change the general pattern of bidirectional GC interactions, although the explained variance was reduced $\left(F_{(2,20)}=7.9, \lambda=0.56, p<0.01\right.$, $\eta^{2}=0.42$; quadratic contrast: $F_{(1,21)}=$ 16.4, $\left.p<0.001, \eta^{2}=0.44\right)$

We next analyzed the mean number of unidirectional GC interactions between all clusters, acknowledging that GC interactions are exclusively unidirectional or bidirectional. Accordingly, we found that overall unidirectional connectivity was larger for neutral pictures $\left(F_{(2,15)}=5.2\right.$, $\lambda=0.59, p<0.05, \eta^{2}=0.41 ;$ quadratic contrast $F_{(1,16)}=8.9, p<0.01, \eta^{2}=0.36$; mean number of unidirectional connections pleasant: $1.18 \pm 0.26$; neutral: $1.65 \pm$ 0.15 ; unpleasant: $0.94 \pm 0.20$ ). Including the six subjects with unreliable GC interactions resulted in no significant differences in unidirectional GC interactions and in a weak tendency for a quadratic contrast $\left(F_{(2,20)}=2.2, \lambda=0.82, p=0.14, \eta^{2}=0.18\right.$; quadratic contrast: $F_{(1,21)}=3.5, p=0.07$, $\eta^{2}=0.14$ ). Although the overall number of unidirectional GC interactions across all clusters was higher for neutral pictures in subjects showing reliable GC interactions, the McNemar test did not reveal significant differences for individual connections (all $p$ values $>0.05$ ).

By contrast, GC interactions associated with viewing pleasant versus neutral pictures revealed a significantly greater proportion of bidirectional connections between right occipitoparietal and prefrontal clusters $(p=0.03$; Fig. $3 B$, left; including all subjects, $p=0.04$ ). The remaining dipole cluster combinations did not show significant GC interaction differences ( $p$ values $>0.11$ ). For unpleasant pictures, bidirectional connectivity between right frontal and occipitoparietal clusters was also significantly greater than that for neutral pictures $(p=0.001$; Fig. $3 B$; including all subjects, $p=0.005)$. Furthermore, greater bidirectional connectivity was observed for this picture type between left and right occipitoparietal clusters $(p=0.01$; Fig. $3 B$; including all subjects, $p=0.04$ ). Left occipitoparietal and right frontal connectivity differences were not observed ( $p=0.57)$. Critically, pleasant and unpleasant picture categories did not show any differential connec-

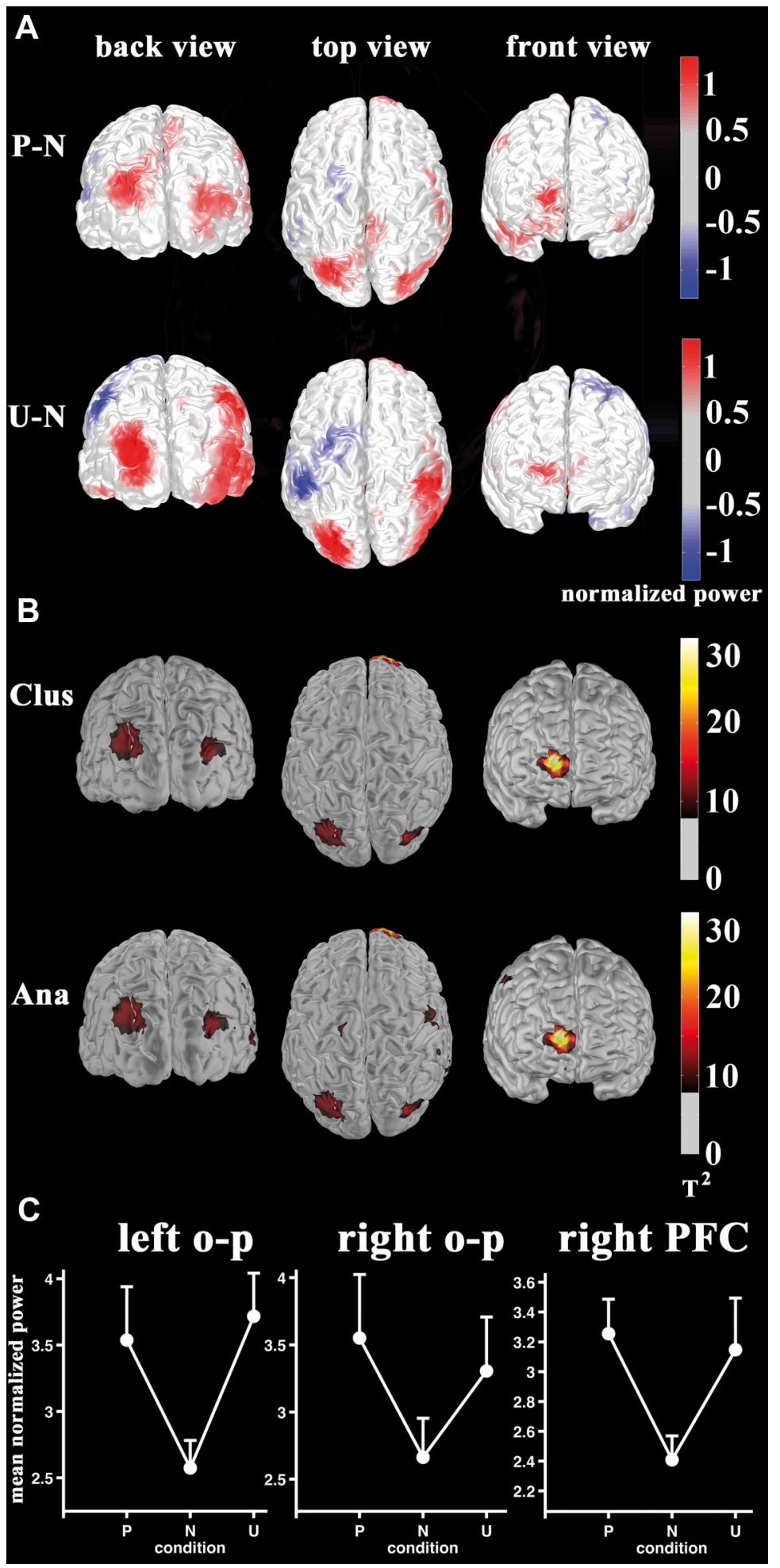

Figure 2. The mLPP arousal modulation is localized to right prefrontal and bilateral occipitoparietal cortex. A, Beamformer difference maps of relative power changes with respect to baseline between pleasant versus neutral (top) and unpleasant versus neutral (bottom) are shown. Color bars indicate normalized power difference. $\boldsymbol{B}$, Statistical maps of the quadratic contrast (pleasant $=$ unpleasant $>$ neutral) in source space based on nonparametric cluster-based permutations (top; threshold $T^{2}>8.0$ corresponding to the smallest $T^{2}$ value across dipole clusters) and analytical multivariate testing (bottom; $T^{2}>7.9$ ) are shown. Color bars indicate multivariate $T^{2}$ values. C, Mean relative power evoked by picture category during the mLPP time window is plotted for each dipole cluster averaged across participants. P, Pleasant; N, neutral; U, unpleasant; Clus, cluster based; Ana, analytical; 0-p, occipitoparietal. 
Table 1. Dipole cluster coordinates in MNI space

\begin{tabular}{lrrrrr}
\hline & \multicolumn{2}{l}{ MNI coordinates } & \\
\cline { 2 - 4 } R0I & $x$ & $y$ & $z$ & Hotelling's $T^{2}$ \\
\hline Right occipital-parietal junction & 32 & -84 & 34 & 12.1 \\
Left occipital-parietal junction & -23 & -83 & 44 & 9.9 \\
Right superior frontal gyrus & 20 & 68 & 12 & 25.9
\end{tabular}

The coordinates represent the center of gravity of the clusters. Note that these values are for informative purposes as Beamformer MEG localization are smoothed and not as exact as fMRI localizations.

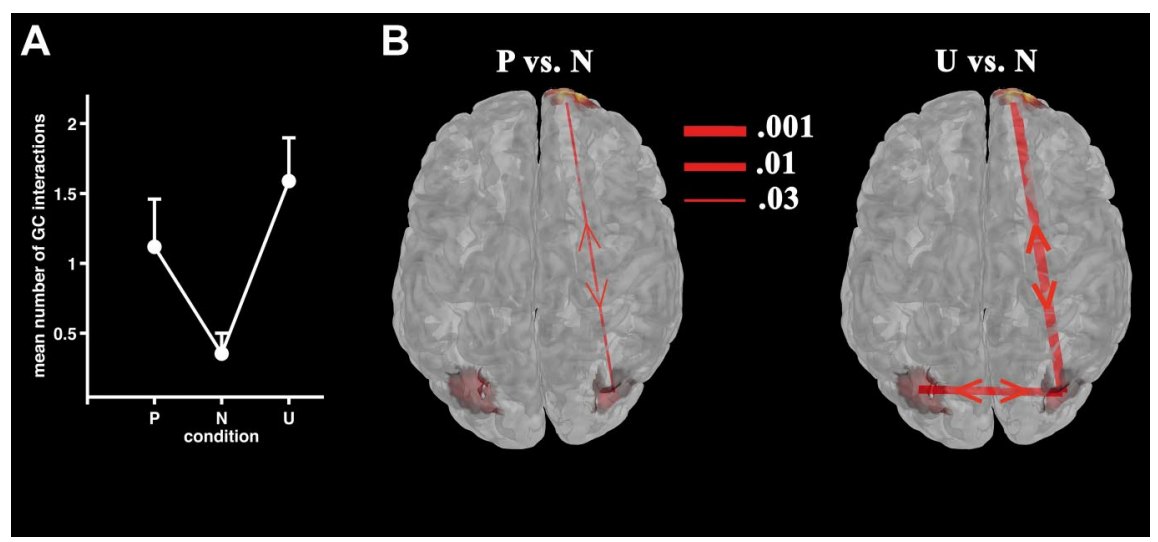

Figure 3. Granger causality modeling of the $\mathrm{mLPP}$ network. $A$, Mean overall number of bidirectional $\mathrm{GC}$ interactions for each picture category averaged across 17 participants. $\boldsymbol{B}$, Significant differences in proportions of bidirectional GC interactions between pleasant $(\mathrm{P})$ (left) and unpleasant $(\mathrm{U})$ versus neutral $(\mathrm{N})$ (right panel) stimuli are illustrated, superimposed on a top view of the statistical map taken from Figure $2 B$. Line thickness indicates the significance level on McNemar testing.

tivity pattern between prefrontal and occipitoparietal brain regions (all $\mathrm{p}$ values $>0.18$ ).

We next characterized the temporal profile of the observed arousal-modulated bidirectional right fronto-occipitoparietal GC interactions. GC interaction values for neutral pictures were subtracted from the averaged pleasant and unpleasant GC values to form arousal-indexed GC values. This was done separately for time intervals $0.2-0.4,0.4-0.6$ (mLPP time window), and $0.6-0.8 \mathrm{~s}$. Figure $4 A$ demonstrates that arousal-indexed GC values were at their maximum during the mLPP time window $\left(F_{(2,15)}=7.1, \lambda=0.51, p<0.01, \eta^{2}=0.48\right.$, linear time contrast: $F_{(1,16)}=2.9, p=0.11, \eta^{2}=0.16$; quadratic time contrast: $F_{(1,16)}$ $=12.8, p<0.01, \eta^{2}=0.45$; single comparisons: interval $0.2-$ $0.4 \mathrm{~s}$ vs interval $0.4-0.6 \mathrm{~s}: p<0.001$; interval $0.4-0.6 \mathrm{~s}$ vs interval $0.6-0.8 \mathrm{~s}: p=0.06$; interval $0.2-0.4 \mathrm{~s}$ vs interval $0.6-0.8 \mathrm{~s}: \mathrm{n}$. s.). Thus, bidirectional right prefrontal-occipitoparietal connectivity is greatest during the mLPP time interval during which all three regions show arousal modulation (Fig. 4B).

\section{Discussion}

Our data provide a neurobiological basis for the late latency response typically observed in response to emotionally arousing stimuli. We first demonstrate, at the level of MEG sensors, a slow magnetic flux drift that shows significant emotional arousal modulation between 0.4 and $0.6 \mathrm{~s}$ after stimulus onset. The striking similarity of the temporal profile of observed ERFs to the LPP observed in ERP studies (Schupp et al., 2000) indicates that this response represents a neuromagnetic counterpart of the electrophysiological LPP (mLPP). Applying beamformer source localization, we then demonstrate that the emotional modulation of the mLPP, observed at the sensor level, is generated in a neuroanatomical network comprising bilateral occipitoparietal and right prefrontal cortex.
That prefrontal cortex is a component of the network giving rise to the $\mathrm{mLPP}$ is strongly supported by patient studies showing that direct prefrontal electrocortical stimulation disrupts the arousal-modulated LPP (Hajcak et al., 2010), and by previous suggestions that the late latency response is under voluntary cognitive control (Hajcak and Nieuwenhuis, 2006; Hajcak et al., 2009). The PFC response observed here is in contrast to the failure of previous ERP studies (Keil et al., 2002; Schupp et al., 2007) to demonstrate prefrontal involvement in LPP generation, which may reflect their use of less accurate source localization techniques and simpler head models than that employed here. These studies (Keil et al., 2002; Schupp et al., 2007) do, however, accord with the current findings in that they localize LPP generation to occipitoparietal areas.

Few fMRI studies have reported emotion-evoked occipitoparietal activation (Lang et al., 1998; Sabatinelli et al., 2007). Activation in this cortical region is more often observed in tasks requiring spatial or feature-based attention (i.e., Fernandez-Duque and Posner, 2001; Slagter et al., 2007). Thus, localizing the mLPP to bilateral occipitoparietal areas is in keeping with a consensus that the LPP reflects an attentional process (Cuthbert et al., 2000) recruited by motivationally salient stimuli. Occipitoparietal activity has also been observed following eye movements (Law et al., 1998). To eschew a potential contribution of eye movements to activity observed in the current study, subjects were instructed to maintain central fixation during picture presentation. Instructions to maintain fixation in the context of the current task has been shown to yield no differences in horizontal or vertical eye movements to emotional versus neutral pictures (Lang et al., 1998). Furthermore, given that saccadic eye movements provoke large biomagnetic fields, by excluding epochs with amplitudes exceeding 3 pT from our analysis we can rule out that eye movements contributed to the observed neuromagnetic signal changes.

It should be noted that the late latency response we report is in contrast to early emotion-evoked neuromagnetic activity observed in ventral extrastriate cortex (Rudrauf et al., 2008) beginning from $0.1 \mathrm{~s}$ poststimulus onset. We do, however, observe, at the sensor level an emotional modulation of the ERFs before the onset of mLPP time window at $0.2-0.4 \mathrm{~s}$ (Fig. $1 A$ ). This ERF modulation most likely reflects volume conduction of the neuromagnetic counterpart of the early posterior negativity known to localize to temporal sensor clusters (Peyk et al., 2008). As the $\mathrm{mEPN}$ is not the focus of our study, we did not investigate this component further. By contrast to effects at the sensor level, the amplitude of arousal modulation within the prefrontaloccipitoparietal network identified by our beamformer analysis is highly specific to the MLPP time window.

A series of studies controlling the psychological factors that influence the arousal-modulated LPP (Codispoti et al., 2006a; Hajcak and Nieuwenhuis, 2006; Hajcak et al., 2007; Schupp et al., 2007; Foti and Hajcak, 2008; Dunning and Hajcak, 2009; Hajcak et al., 2009) together suggest that this response reflects a combination of "bottom-up" and "top-down" processing (Ferrari et al., 2008). Localizing the cortical generators of the mLPP to frontal 
A

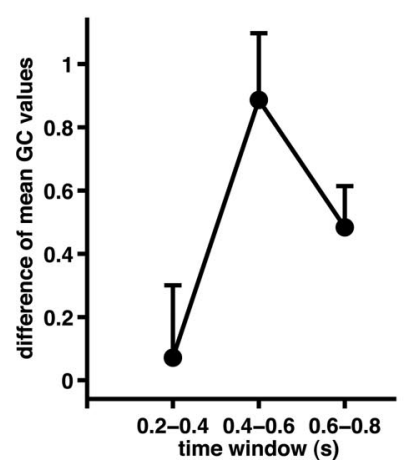

B

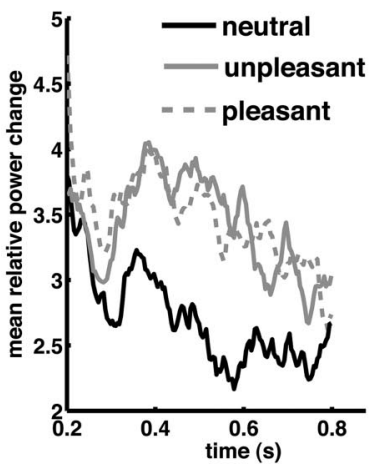

right o-p

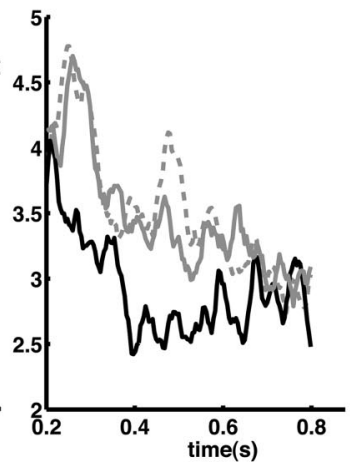

right PFC

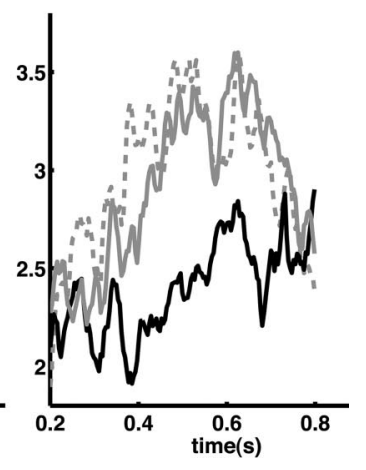

Figure 4. Arousal indexed bidirectional Granger causality values are maximal during the $\mathrm{mLPP}$ temporal window. $\boldsymbol{A}$, Difference (high vs low arousing) of mean bidirectional GC values between right prefrontal and occipitoparietal cortical clusters are plotted for the time intervals of interest. $\boldsymbol{B}$, Mean relative source power waveforms from each $\mathrm{mLPP}$ dipole cluster are plotted for each picture category. 0-p, 0ccipitoparietal.

and posterior regions raises the possibility that this network furnishes these putative "bottom-up" and "top-down" mechanisms. Testing for GC interactions between the reconstructed time series of the three cortical clusters in our network revealed enhanced bidirectional connectivity between right prefrontal and occipitoparietal cortex for high-arousing emotional (pleasant and unpleasant) versus neutral pictures. The magnitude of this bidirectional GC effect is at its maximum in the mLPP time window $(0.4-0.6 \mathrm{~s})$. This indicates that the prefrontal-occipitoparietal coupling is not present throughout picture viewing; hence, is not simply related to task demands but reflects a process intimately related with mLPP generation.

Pleasant pictures relative to neutral ones induce less significant differences in GC interactions compared to unpleasant versus neutral stimuli. As pleasant and unpleasant pictures evoke equally strong source activity in the prefrontal-occipitoparietal brain network (Fig. 2C), it is unlikely that this effect is due to differential signal power across conditions. Instead we suggest that stronger prefrontal-occipitoparietal coupling for unpleasant stimuli reflects the fact that these pictures were rated as more arousing than pleasant ones.

The $\mathrm{mLPP} / \mathrm{LPP}$ represents a slow going field/potential shift between 400 and $600 \mathrm{~ms}$. However, studies using longer picture presentation times report LPP responses up to several seconds even after stimulus offset (Hajcak and Olvet, 2008). Such long responses would be in keeping with sustained network activity. For example, steady-state visual-evoked potentials (ssVEPs), which represent sustained oscillatory cortical responses (Moratti et al., 2007) and can be modulated by emotion (Keil et al., 2003; Moratti et al., 2004), are thought to be driven by re-entrant connections from parietal to visual cortex (Keil et al., 2009). However, ssVEPs represent activity at early stimulus processing stages. Critical for our late latency mLPP observations, reverberating activity in a prefrontal-parietal network is one hypothesized mechanism for sustained neuronal responses at later stages of stimulus processing (Wang, 2001). Alternatively, the process mediating the sustained temporal profile of the mLPP/ LPP could result from local activity in any of our observed clusters (Wang, 2001). However, the fact that direct electrocortical inhibition of prefrontal cortex leads to decreased LPP in posterior sites (Hajcak et al., 2010) argues for a network mechanism in the generation of this late latency neuronal response. This explanation is consistent with the observation that engagement of prefrontal cortex by increasing load in a concurrent working memory task also reduces LPP amplitude in response to aversive pictures (Macnamara et al., 2011). Thus, the mLPP/ LPP likely represents sustained network activity in anterior-posterior brain regions as reflected by our finding of bidirectional prefrontaloccipitoparietal coupling.

The observed reciprocal codependency between frontal and posterior regions may therefore represent the neurobiological mechanism underlying the proposal, derived from psychological manipulations, that the LPP represents both "bottom-up" and "top-down" processes. However, this suggestion must be viewed within the limitations of our analysis. Granger causality tests for a statistical relationship, based on temporal precedence (Seth, 2010), among neuromagnetic time courses in prefrontal and occipitoparietal clusters. Importantly, identifying bidirectionality of prefrontal-occipitoparietal functional connectivity, indexed by GC, is not equivalent to identifying biophysical causal interactions between these brain areas. It is possible, therefore, that a third latent variable, not detected by our source analysis, may have caused the observed GC interactions. For example, we cannot rule out the participation of the amygdala in the emotion network we describe. The amygdala has a closed field neuronal structure; thus, is technically difficult to map with MEG/EEG.

An alternative approach to elucidating an amygdala role in generating late neurophysiological responses is to measure ERPs/ ERFs in patients with amygdala damage. For example, Rotshtein et al. (2010) reported late ERPs in response to aversive face stimuli in control subjects that were reduced in patients with amygdala lesions. However, emotion-induced ERPs in this study (Rotshtein et al., 2010) showed a phasic response between 550 and $600 \mathrm{~ms}$ and hence did not share the slow, sustained properties of the LPP. This difference may reflect the presentation of face stimuli as opposed to emotional scenes typically employed in previous studies on the LPP. Thus, although the amygdala is implicated in generating a late response to emotion, the absence of an LPP in this previous study (Rotshtein et al., 2010) means that this cannot be taken as evidence for amygdala involvement in modulating the prefrontal-occipitoparietal mLPP network we describe. Furthermore, a critical argument against inclusion of the amygdala in the LPP-generating network is that amygdala responses undergo rapid habituation to repeated presentations of emotional stimuli (Breiter et al., 1996), whereas LPP emotion effects are resistant to habituation (Codispoti et al., 2006a). 
In contrast to Granger causality, dynamic causal modeling (DCM) (Friston et al., 2003, 2011), another approach to understanding brain connectivity, employs an explicit generative model of how observed data are caused, thus enabling measurement of effective connectivity. However, DCM requires a priori knowledge of a specific biophysical model. Given that this is the first demonstration of arousal-modulated late latency prefrontal activity and that it forms part of a prefrontal-occipitoparietal network, we therefore elected the simpler GC analysis over DCM (Friston, 2009), acknowledging that we report directed functional connectivity and not effective connectivity.

In summary, we applied high temporal resolution MEG recordings combined with recently developed source modeling techniques to determine the underlying cortical sources of the late latency response to emotion. Employing measures of directed functional connectivity, we demonstrate that the combination of top-down and bottom-up functions ascribed to this late latency response may originate in bidirectional prefrontaloccipitoparietal codependency, which parallels the time course of the $\mathrm{mLPP} / \mathrm{LPP}$. Given that the LPP is abnormal in a number of psychiatric conditions, such as generalized anxiety disorder (MacNamara and Hajcak, 2009, 2010; Macnamara et al., 2011) and schizophrenia (Horan et al., 2010), knowledge of the neurobiological mechanism underlying the LPP can, in turn, inform the pathological basis of these conditions.

\section{References}

Bradley MM, Hamby S, Löw A, Lang PJ (2007) Brain potentials in perception: picture complexity and emotional arousal. Psychophysiology 44:364-373.

Breiter HC, Etcoff NL, Whalen PJ, Kennedy WA, Rauch SL, Buckner RL, Strauss MM, Hyman SE, Rosen BR (1996) Response and habituation of the human amygdala during visual processing of facial expression. Neuron 17:875-887.

Codispoti M, Ferrari V, Bradley MM (2006a) Repetitive picture processing: autonomic and cortical correlates. Brain Res 1068:213-220.

Codispoti M, Ferrari V, De Cesarei A, Cardinale R (2006b) Implicit and explicit categorization of natural scenes. Prog Brain Res 156:53-65.

Collins DL, Zijdenbos AP, Kollokian V, Sled JG, Kabani NJ, Holmes CJ, Evans AC (1998) Design and construction of a realistic digital brain phantom. IEEE Trans Med Imaging 17:463-468.

Cuthbert BN, Schupp HT, Bradley MM, Birbaumer N, Lang PJ (2000) Brain potentials in affective picture processing: covariation with autonomic arousal and affective report. Biol Psychol 52:95-111.

Dolcos F, Cabeza R (2002) Event-related potentials of emotional memory: encoding pleasant, unpleasant, and neutral pictures. Cogn Affect Behav Neurosci 2:252-263.

Dunning JP, Hajcak G (2009) See no evil: directing visual attention within unpleasant images modulates the electrocortical response. Psychophysiology 46:28-33.

Fernandez-Duque D, Posner MI (2001) Brain imaging of attentional networks in normal and pathological states. J Clin Exp Neuropsychol 23:74-93.

Ferrari V, Codispoti M, Cardinale R, Bradley MM (2008) Directed and motivated attention during processing of natural scenes. J Cogn Neurosci 20:1753-1761.

Foti D, Hajcak G (2008) Deconstructing reappraisal: descriptions preceding arousing pictures modulate the subsequent neural response. J Cogn Neurosci 20:977-988.

Friston K (2009) Causal modelling and brain connectivity in functional magnetic resonance imaging. PLoS Biol 7:e33.

Friston KJ, Harrison L, Penny W (2003) Dynamic causal modelling. Neuroimage 19:1273-1302.

Friston KJ, Li B, Daunizeau J, Stephan KE (2011) Network discovery with DCM. Neuroimage 56:1202-1221.

Granger C (1969) Investigating causal relations by econometrc models and cross-spectral methods. Econometrica 37:424-438.

Hajcak G, Nieuwenhuis S (2006) Reappraisal modulates the electrocortical response to unpleasant pictures. Cogn Affect Behav Neurosci 6:291-297.
Hajcak G, Olvet DM (2008) The persistence of attention to emotion: brain potentials during and after picture presentation. Emotion 8:250-255.

Hajcak G, Dunning JP, Foti D (2007) Neural response to emotional pictures is unaffected by concurrent task difficulty: an event-related potential study. Behav Neurosci 121:1156-1162.

Hajcak G, Dunning JP, Foti D (2009) Motivated and controlled attention to emotion: time-course of the late positive potential. Clin Neurophysiol 120:505-510.

Hajcak G, Anderson BS, Arana A, Borckardt J, Takacs I, George MS, Nahas Z (2010) Dorsolateral prefrontal cortex stimulation modulates electrocortical measures of visual attention: evidence from direct bilateral epidural cortical stimulation in treatment-resistant mood disorder. Neuroscience 170:281-288.

Horan WP, Wynn JK, Kring AM, Simons RF, Green MF (2010) Electrophysiological correlates of emotional responding in schizophrenia. J Abnorm Psychol 119:18-30.

Huang MX, Mosher JC, Leahy RM (1999) A sensor-weighted overlappingsphere head model and exhaustive head model comparison for MEG. Phys Med Biol 44:423-440.

Keil A, Bradley MM, Hauk O, Rockstroh B, Elbert T, Lang PJ (2002) Largescale neural correlates of affective picture processing. Psychophysiology 39:641-649.

Keil A, Gruber T, Müller MM, Moratti S, Stolarova M, Bradley MM, Lang PJ (2003) Early modulation of visual perception by emotional arousal: evidence from steady-state visual evoked brain potentials. Cogn Affect Behav Neurosci 3:195-206.

Keil A, Sabatinelli D, Ding M, Lang PJ, Ihssen N, Heim S (2009) Re-entrant projections modulate visual cortex in affective perception: evidence from Granger causality analysis. Hum Brain Mapp 30:532-540.

Knösche TR (2002) Transformation of whole-head MEG recordings between different sensor positions. Biomed Tech (Berl) 47:59-62.

Kwiatkowski D, Phillips PC, Schmidt P, Shin Y (1992) Testing the null hypothesis of stationarity against the alternative of a unit root. J Econometrics 54:159-178.

Lang PJ, Bradley MM, Fitzsimmons JR, Cuthbert BN, Scott JD, Moulder B, Nangia V (1998) Emotional arousal and activation of the visual cortex: an fMRI analysis. Psychophysiology 35:199-210.

Lang PJ, Bradley MM, Cuthbert BN (2005) International affective picture system (IAPS): affective ratings of pictures and instruction manual (Technical Report A-6). Gainesville, FL: University of Florida.

Law I, Svarer C, Rostrup E, Paulson OB (1998) Parieto-occipital cortex activation during self-generated eye movements in the dark. Brain 121:2189-2200.

MacNamara A, Hajcak G (2009) Anxiety and spatial attention moderate the electrocortical response to aversive pictures. Neuropsychologia 47:2975-2980.

MacNamara A, Hajcak G (2010) Distinct electrocortical and behavioral evidence for increased attention to threat in generalized anxiety disorder. Depress Anxiety 27:234-243.

Macnamara A, Ferri J, Hajcak G (2011) Working memory load reduces the late positive potential and this effect is attenuated with increasing anxiety. Cogn Affect Behav Neurosci 11:321-331.

Maris E, Oostenveld R (2007) Nonparametric statistical testing of EEG- and MEG-data. J Neurosci Methods 164:177-190.

McNemar Q (1947) Note on the sampling error of the difference between correlated proportions or percentages. Psychometrika 12:153-157.

Moratti S, Keil A, Stolarova M (2004) Motivated attention in emotional picture processing is reflected by activity modulation in cortical attention networks. Neuroimage 21:954-964.

Moratti S, Clementz BA, Gao Y, Ortiz T, Keil A (2007) Neural mechanisms of evoked oscillations: Stability and interaction with transient events. Hum Brain Mapp 28:1318-1333.

Nichols TE, Holmes AP (2002) Nonparametric permutation tests for functional neuroimaging: a primer with examples. Hum Brain Mapp 15:1-25.

Oldfield RC (1971) The assessment and analysis of handedness: The Edinbourgh Inventory. Neuropsychologia 9:97-113.

Oostenveld R, Fries P, Maris E, Schoffelen JM (2011) FieldTrip: open source software for advanced analysis of MEG, EEG, and invasive electrophysiological data. Comput Intell Neurosci 2011:156869.

Peyk P, Schupp HT, Elbert T, Junghöfer M (2008) Emotion processing in the visual brain: a MEG analysis. Brain Topogr 20:205-215.

Phan KL, Wager T, Taylor SF, Liberzon I (2002) Functional neuroanatomy 
of emotion: a meta-analysis of emotion activation studies in PET and fMRI. Neuroimage 16:331-348.

Poline JB, Worsley KJ, Evans AC, Friston KJ (1997) Combining spatial extent and peak intensity to test for activations in functional imaging. Neuroimage 5:83-96.

Rotshtein P, Richardson MP, Winston JS, Kiebel SJ, Vuilleumier P, Eimer M, Driver J, Dolan RJ (2010) Amygdala damage affects event-related potentials for fearful faces at specific time windows. Hum Brain Mapp 31:1089-1105.

Rudrauf D, David O, Lachaux JP, Kovach CK, Martinerie J, Renault B, Damasio A (2008) Rapid interactions between the ventral visual stream and emotion-related structures rely on a two-pathway architecture. J Neurosci 28:2793-2803.

Sabatinelli D, Lang PJ, Keil A, Bradley MM (2007) Emotional perception: correlation of functional MRI and event-related potentials. Cereb Cortex 17:1085-1091.

Sato JR, Fujita A, Cardoso EF, Thomaz CE, Brammer MJ, Amaro E Jr (2010) Analyzing the connectivity between regions of interest: an approach based on cluster Granger causality for fMRI data analysis. Neuroimage 52:1444-1455.
Schupp HT, Cuthbert BN, Bradley MM, Cacioppo JT, Ito T, Lang PJ (2000) Affective picture processing: the late positive potential is modulated by motivational relevance. Psychophysiology 37:257-261.

Schupp HT, Stockburger J, Codispoti M, Junghöfer M, Weike AI, Hamm AO (2007) Selective visual attention to emotion. J Neurosci 27:1082-1089.

Schwarz G (1978) Estimating the dimension of a model. Ann Stat 6:461-464.

Seth AK (2010) A MATLAB toolbox for Granger causal connectivity analysis. J Neurosci Methods 186:262-273.

Slagter HA, Giesbrecht B, Kok A, Weissman DH, Kenemans JL, Woldorff MG, Mangun GR (2007) fMRI evidence for both generalized and specialized components of attentional control. Brain Res 1177:90-102.

Van Veen BD, van Drongelen W, Yuchtman M, Suzuki A (1997) Localization of brain electrical activity via linearly constrained minimum variance spatial filtering. IEEE Trans Biomed Eng 44:867-880.

Wang XJ (2001) Synaptic reverberation underlying mnemonic persistent activity. Trends Neurosci 24:455-463.

Zhou Z, Ding M, Chen Y, Wright P, Lu Z, Liu Y (2009) Detecting directional influence in fMRI connectivity analysis using PCA-based Granger causality. Brain Res 1289:22-29. 